\title{
Increased vulnerability of pre-existing atherosclerosis in ApoE-deficient mice following adenovirus-mediated Fas ligand gene transfer
}

\author{
A. Susanne M. Zadelaar ${ }^{a, b, *}$, Jan H. von der Thüsen ${ }^{c}$, Lianne S. M. Boesten ${ }^{a, b}$, \\ Rob C. Hoeben ${ }^{\mathrm{d}}$, Mark M. Kockx ${ }^{\mathrm{e}}$, Marjan A. Versnel ${ }^{\mathrm{f}}$, Theo J.C. van Berkel ${ }^{\mathrm{c}}$, \\ Louis M. Havekes ${ }^{\mathrm{a}, \mathrm{b}}$, Erik A. L. Biessen ${ }^{\mathrm{c}}$, Bart J.M. van Vlijmen ${ }^{\mathrm{a}, \mathrm{b}}$ \\ ${ }^{a}$ Department of Cardiology, Leiden University Medical Center clo TNO Prevention and Health, Gaubius Laboratory, \\ Zernikedreef 9, P.O. Box 2215, 2301 CE Leiden, The Netherlands \\ b TNO-PG/Gaubius Laboratory, Leiden, Zernikedreef 9, P.O. Box 2215, 2301 CE Leiden, The Netherlands \\ ${ }^{c}$ Division of Biopharmaceutics, Leiden/Amsterdam Center for Drug Research, Leiden, The Netherlands \\ ${ }^{\mathrm{d}}$ Department of Molecular Cell Biology, Leiden University Medical Center, Leiden, The Netherlands \\ ${ }^{\mathrm{e}}$ Department of Pathology, Middelheim Academic Hospital, Antwerp, Belgium \\ ${ }^{\mathrm{f}}$ Department of Immunology, Erasmus Medical Center, Rotterdam, The Netherlands
}

Received 15 December 2004; received in revised form 4 March 2005; accepted 14 March 2005

Available online 31 May 2005

\begin{abstract}
Objective: The death receptor Fas and Fas ligand (FasL) are present in human advanced atherosclerotic plaques. The activation of the Fas/FasL pathway of apoptosis has been implicated in plaque vulnerability. In the present study, we investigated whether overexpression of FasL in pre-existing atherosclerotic lesions can induce lesion remodelling and rupture-related events.

Methods and results: Carotid atherogenesis was initiated in apolipoprotein E-deficient mice by placement of a perivascular silastic collar. The resulting plaques were incubated transluminally with recombinant adenovirus carrying FasL (Ad-FasL, lateral) or control $\beta$-galactosidase (Ad-LacZ, contralateral). Transfection was restricted to the smooth muscle cell-rich cap of the plaque, and FasL expression led to a three-fold increase in apoptosis in the cap one day after gene transfer. Three days after gene transfer, FasL expression led to a $38 \%$ reduction in the number of cap cells. Two weeks after Ad-FasL transfer, non-thrombotic rupture, intra-plaque haemorrhage, buried caps and iron deposits were observed in 6 out of 17 Ad-FasL-treated carotid arteries versus 0 out of 17 controls $(P=0.009)$, indicative of enhanced plaque vulnerability. Conclusions: These data demonstrate that advanced murine plaques are sensitive to Fas/FasL-induced apoptosis, which may indicate that stimulation of this pathway could result in plaque remodelling towards a more vulnerable phenotype.
\end{abstract}

(C) 2005 Published by Elsevier Ireland Ltd.

Keywords: Apoptosis; Atherosclerosis; Carotid arteries; Gene expression; Vascular smooth muscle

\section{Introduction}

Fas is one of the major apoptosis-mediating receptors from the tumor necrosis factor- $\alpha$-receptor $(\mathrm{TNF} \alpha-\mathrm{R})$ superfamily. When Fas ligand (FasL) binds to Fas, programmed cell death is rapidly induced. The Fas/FasL pathway is involved in tissue homeostasis, the down-regulation of immune reactions

\footnotetext{
* Corresponding author. Tel.: +31 71 5181396; fax: +31 715181904 .

E-mail address: asm.zadelaar@pg.tno.nl (A.S.M. Zadelaar).
}

and $\mathrm{T}$ cell mediated toxicity [1]. Additionally, FasL has a gatekeeper function on endothelial cells (ECs) of the vessel wall [2]. The death receptor Fas and FasL are present in human advanced atherosclerotic plaques and the activation of the Fas/FasL pathway of apoptosis has been implicated in plaque remodelling [3-5].

In the atherosclerotic plaque, FasL is present on $\mathrm{T}$ cells and macrophages, and on ECs forming the outermost layer of the fibrous cap and the plaque [3-5]. The Fas receptor is present on ECs, smooth muscle cells (SMCs) and macrophages [6,7], 
coinciding with evidence of apoptosis in the shoulder area $[6,8]$. In vitro studies indicate that several factors in an atheromatous setting, including macrophage-derived cytokines and p53, cause translocation or trafficking of Fas to the cell surface of SMCs [6,9]. In addition to Fas, in macrophages FasL is also transported to the cell surface in response to oxidized low-density lipoprotein (Ox-LDL) [6,10]. The presence of Fas and FasL in human atherosclerotic plaques [3,6], as well as the fact that human blood-derived macrophages can induce apoptosis in human plaque-derived SMCs by Fas/FasL interactions in vitro [11], have fuelled speculation about the role of the Fas/FasL pathway of apoptosis in lesion remodelling.

A series of previous in vivo studies focussed on the role of FasL in vascular disease at the level of the normal, ballooninjured, or denuded vessel wall, either in a normo- or hypercholesterolemic setting [12-14]. These studies demonstrated that amongst others adenovirus-delivered FasL can modulate infiltration of inflammatory cells and thereby the initiation and progression of atherosclerosis. In the present in vivo study, and in contrast to previous studies, we focused on the role of FasL in pre-existing advanced atherosclerotic plaques; a setting in which cells may be highly sensitive to this apoptotic trigger. To this end, we transduced advanced collarinduced [15] pre-existing carotid artery lesions in ApoE-/mice with adenovirus carrying a murine FasL transgene.

The present study shows that advanced murine plaques are sensitive to Fas/FasL-induced apoptosis, which may indicate that stimulation of this pathway could result in plaque remodelling towards a more vulnerable phenotype.

\section{Methods}

\subsection{Adenoviral vectors}

Serotype 5 adenoviral vectors containing the murine Fas ligand cDNA (Ad-FasL; kind gift from Dr. K Walsh, Molecular Cardiology, Whitaker Cardiovascular Institute, Boston, USA) or bacterial $\beta$-galactosidase cDNA (Ad-LacZ) driven by the cytomegalovirus promoter were used and have been described previously [13]. Adenoviral vectors were propagated in PER.C6 cells, tested and their titer quantified according to standard protocols [16].

\subsection{Carotid collar placement and adenovirus injection}

Carotid atherosclerotic lesions were induced by perivascular collar placement in male ApoE-/- mice, aged 10-12 weeks [15]. Four weeks after surgery, the collars were removed and $10 \mu$ l of adenoviral suspension at $1.5 \times 10^{10} \mathrm{pfu} / \mathrm{ml}$ was instilled bilaterally (at random 1 side with Ad5-CMV.FasL, the contralateral side with Ad5CMV.LacZ) into the common carotid artery via the external carotid as described [17]. $1(n=9), 3(n=9)$ or 14 days $(n=17)$ after transfection mice were sacrificed by perfusion with phosphate buffered saline and tissues were perfusion fixed using phosphate buffered formalin $\mathrm{pH}$ 7.4. The regulatory authority of the institutional experimental animal committee approved all animal work.

\subsection{Tissue preparation and histological analysis}

The obtained carotid artery specimens were either dehydrated and embedded in paraffin or snap-frozen to prepare cryosections. Transverse $5 \mu \mathrm{m}$ cross-sections were prepared, serially mounted, and routinely stained with hematoxylin (Merck Diagnostica, Darmstadt, Germany) and eosin (HE) (Sigma), Masson's trichrome (kit \#HT15, Sigma Diagnostics, St. Louis, USA) and picrosirius red (Chroma, Stuttgart, Germany). $\beta$-Galactosidase was demonstrated by incubation with X-gal $\left(1 \mathrm{mg} / \mathrm{ml}\right.$, Sigma) at $37^{\circ} \mathrm{C}$ for $4 \mathrm{~h}$. Apoptosis was assessed by terminal deoxynucleotidyl transferase endlabeling (TUNEL) $[18,19]$. Iron deposits were visualized by Perls iron staining.

Slides were stained with an antibody against Fas [20]. Serial slides were stained with antibodies against the endothelial cell marker CD31 (dilution 1:200, Pharmingen), SM- $\alpha$ actin (clone 1A4, dilution 1:1500, DAKO) and the monocyte/macrophage antibody AIA31240 (dilution 1:3000, Accurate Chemical and Scientific, New York, USA). Polyclonal biotinylated goat anti-rat Ig (1:100, Pharmingen), biotinylated rabbit anti-mouse Ig (1:300, DAKO), goat anti-mouse IgG peroxidase conjugate (dilution 1:500, Nordic, Tilburg, The Netherlands) and donkey anti-rabbit Ig (1:3000, Amersham) were used as secondary antibodies. Following incubation with horseradish peroxidase labeled avidin-biotin complex (abc/HRP) (DAKO), peroxidase activity was visualised using NovaRED (Vector) or 3,3'-diamino-benzidine as the substrate.

Morphometrical analysis (media area, total intima area, fibrocellular cap area, core area, nuclei counts and ratios) of three sections per carotid artery (point of maximum stenosis) at $80 \mu \mathrm{m}$ intervals was blindly performed using LeicaQwin software (Leica Imaging Systems, Cambridge, UK), as described previously [17].

\subsection{Statistical analysis}

All data are represented as mean \pm S.D. Data were analysed using the non-parametric Mann-Whitney rank sum test. Frequency data analysis was carried out by Fisher's exact test. $P$-values less than 0.05 were regarded as statistically significant.

\section{Results}

\subsection{Adenoviral activity}

Functionality of the transgene was confirmed by intravenous injection of both LacZ and FasL constructs $\left(2 \times 10^{9} \mathrm{pfu} / \mathrm{mouse}\right)$ in wild type animals and Fas-defective 
mice (lpr mice). As analysed on $5 \mu \mathrm{m}$ liver cryosections, three days after intravenous injection of Ad-LacZ, virtually all liver cells of both wild type and $l p r$ mice displayed $\beta$ galactosidase activity (not shown). Following Ad-FasL injection, hematoxylin-eosin stained paraffin sections of livers of wild type mice displayed the typical features of apoptosis, including cell shrinkage, nuclear condensation and fragmentation in $75.8 \pm 5.1 \%$ of the hepatic cells ( $n=3$, not shown). Liver apoptosis in Ad-FasL injected mice was accompanied by extremely high serum alanine aminotransferase (ALT) levels (>10,000 U/L versus $42 \pm 8 \mathrm{U} / \mathrm{ml}$ for Ad-LacZ). In contrast, Ad-FasL injected $l p r$ mice displayed no features of liver apoptosis and damage (serum ALT levels of $34 \pm 6 \mathrm{U} / \mathrm{L}$, $n=3$ ). This demonstrates that the two adenoviral constructs have comparable transduction efficiency, with Ad-FasL being a potent and specific inducer of apoptosis of Fas-bearing liver cells.

\subsection{Effects of Ad-FasL one day after transfection}

Presence of the Fas death receptor in pre-existing carotid artery lesions of ApoE-deficient mice was confirmed by immunohistochemistry. The cap area demonstrates the presence of Fas-positive lining of morphologically identified SMCs (Fig. 1B), which was comparable with Fas-positive lining observed for hepatocytes (Fig. 1D). In addition, Fas positivity was observed for endothelial cells and (core) macrophages.

Previous local adenoviral delivery to pre-existing collarinduced carotid artery lesions of ApoE-deficient mice resulted in efficient transduction of a superficial layer of the SM- $\alpha$-actin-positive fibrous cap [17]. Endothelial cells and occasional superficial macrophages can also be targeted by this procedure. In the present study, we confirmed transfection of the SMC-rich cap area by $\beta$-galactosidase staining of a collar-induced carotid artery lesion one day after incuba-

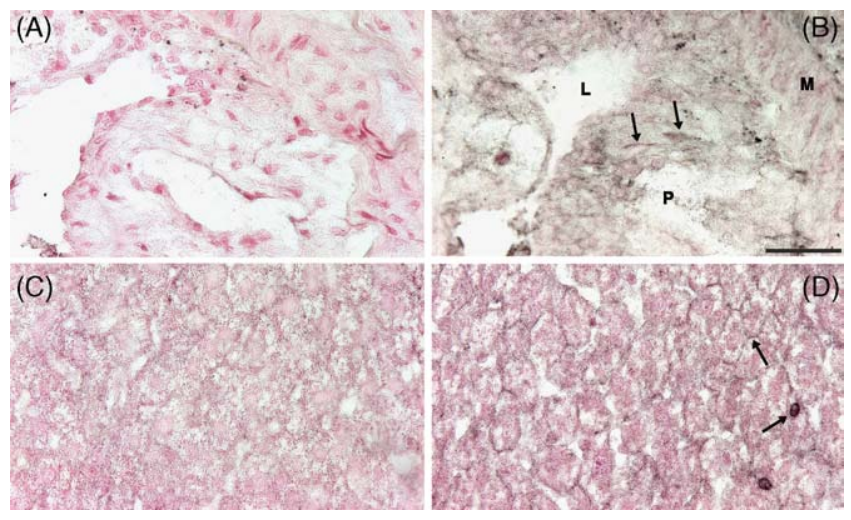

Fig. 1. Immunohistochemistry of the Fas receptor. (A) Control IgG antibody and (B) Fas antibody staining of atherosclerotic plaque, demonstrating that amongst others SMCs have Fas-positive lining (B, arrows). (C) Control IgG antibody and (D) Fas antibody staining of wild type liver, showing Fas-positive lining of liver cell membranes and leukocytes (D, arrows). (M, media, $\mathrm{P}$, plaque, L, lumen, magnification $400 \times$.)

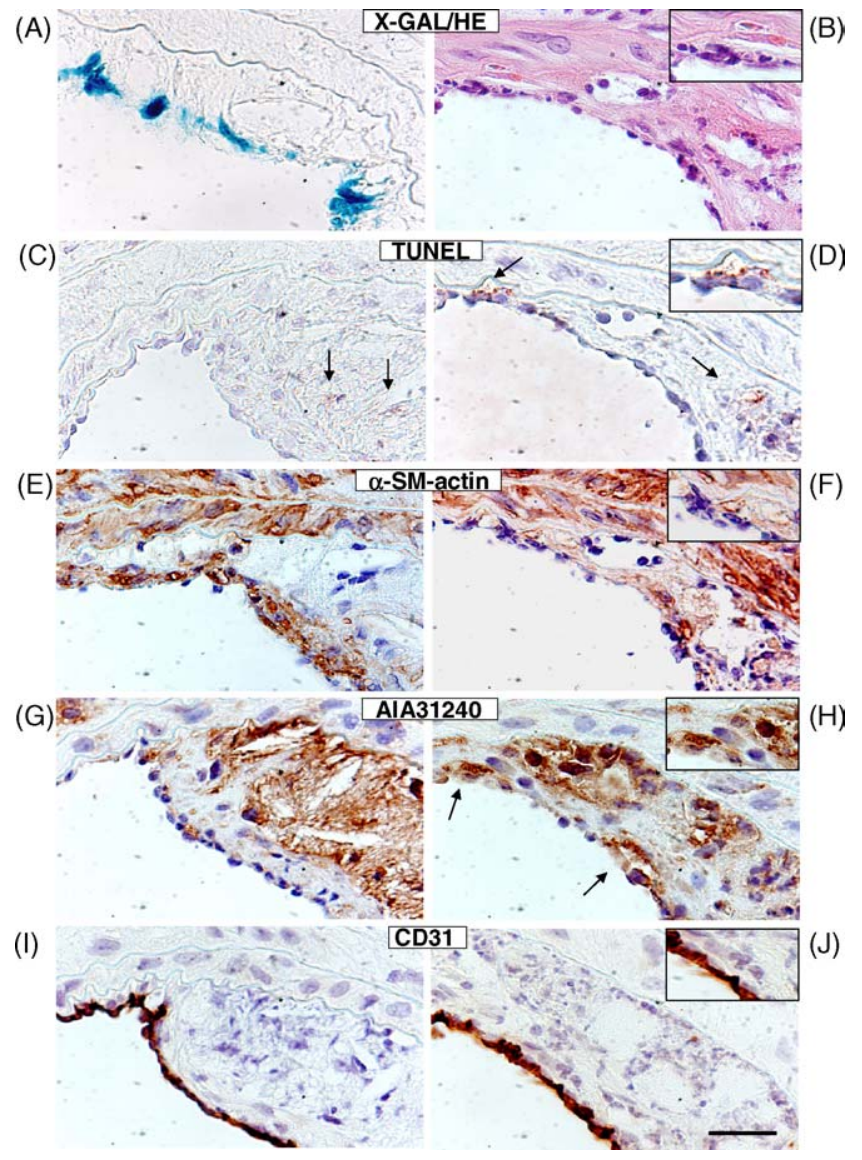

Fig. 2. (Immuno)histochemical staining of collar-induced carotid artery lesions one day after transfection of Ad-LacZ (left panels) and Ad-FasL (right panels). (A) X-gal staining, showing an Ad-LacZ-incubated carotid artery lesion three days after transfection and (B) hematoxylin and eosin staining one day after Ad-FasL incubation. (C and D) TUNEL staining for apoptosis. Apoptosis is observed as positive red staining. (E and F) SM- $\alpha$-actin staining. ( $\mathrm{G}$ and $\mathrm{H}$ ) Monocyte/macrophage AIA31240 staining. (I and J) CD31 staining (magnification $400 \times$; scale bar $25 \mu \mathrm{m}$, insets magnification $600 \times$ ).

tion with control Ad-LacZ, showing $\beta$-galactosidase-positive blue cells in the shoulders and cap of the lesion (Fig. 2A).

Success of transfection by Ad-FasL was monitored using TUNEL staining, which allows detection of FasL-induced apoptosis. One day after transfection, TUNEL-positive nuclei were absent $100 \mu \mathrm{m}$ proximal to the atherosclerotic lesion at the level of the non-diseased vessel wall, which was also exposed to either Ad-FasL (lateral) or Ad-LacZ (contralateral) (Table 1). By contrast, at the site of the atherosclerotic lesion, Ad-FasL incubation resulted in the presence of TUNELpositive nuclei, located in the SM- $\alpha$-actin-positive cap area, as well as in the core (Fig. 2D). In Ad-LacZ-incubated lesions such TUNEL-positive material was mainly confined to the core of the lesions and only incidentally found in caps of lesions (Fig. 2C). Quantitation of the number of TUNELpositive nuclei in the core showed equal presence of TUNELpositive nuclei for Ad-FasL and Ad-LacZ-incubated lesions $(3.3 \pm 4.9$ versus $4.0 \pm 2.3 \%$ TUNEL-positive nuclei for AdLacZ and Ad-FasL, respectively, Table 1). Quantification of 
Table 1

Quantification of TUNEL-positive nuclei and the number of AIA31240positive cells present at the lumenal side of the endothelial lining of Ad-FasLtreated or contralateral Ad-LacZ-treated pre-existing carotid atherosclerotic plaques and normal vessel wall one day after transfection

\begin{tabular}{lcc}
\hline & Ad-LacZ & Ad-FasL \\
\hline $\begin{array}{l}\text { TUNEL-positive nuclei (\% of total nuclei) } \\
\text { Non-diseased vessel wall }\end{array}$ & $0.0 \pm 0.0$ & $0.0 \pm 0.0$ \\
Atherosclerotic vessel wall & $3.7 \pm 2.0$ & $5.5 \pm 1.8$ \\
$\quad$ Total & $3.3 \pm 4.9$ & $4.0 \pm 2.3$ \\
Core & $0.5 \pm 0.7$ & $1.5 \pm 1.7^{\mathrm{a}}$ \\
Cap & $1.7 \pm 1.3$ & $2.5 \pm 1.1$ \\
Adhering lumenal AIA31240-positive cells (cells/section) \\
Non-diseased vessel wall & $1.2 \pm 0.9$ & $10.2 \pm 3.9^{\mathrm{a}}$ \\
Atherosclerotic vessel wall &
\end{tabular}

a Significantly different from Ad-LacZ-treated group $(P<0.05)$.

the number of TUNEL-positive nuclei in the cap revealed that Ad-FasL-incubated lesions had a significant $(P=0.04)$ threefold higher presence of TUNEL-positive nuclei as compared to the Ad-LacZ-incubated lesions $(0.5 \pm 0.7$ versus $1.5 \pm 1.7$ TUNEL-positive nuclei for Ad-LacZ and Ad-FasL, respectively, Table 1). This indicates that Ad-FasL induces apoptosis not only in the liver of systemically-treated animals, but also specifically at the level of the atherosclerotic vessel wall of locally-treated animals.

The increase of TUNEL positivity in cap cells of the AdFasL-incubated lesions coincided with increased presence of cells staining positive for monocyte/macrophage antibody AIA31240, both subendothelial and at the lumenal side of the endothelial lining (Fig. 2H). Quantitation of the AIA31240positive cells at lumenal side of the endothelial lining yielded $10.2 \pm 3.9$ compared to $1.2 \pm 0.9$ cells per section $(n=4)$ for Ad-LacZ-incubated lesions $(P=0.01$, Table 1). Although the presence of AIA31240-positive cells was also detected at the level of the adenovirus-incubated non-diseased part of the vessel wall, their number was low and comparable for Ad-LacZ and Ad-FasL incubations (2.5 \pm 1.1 versus $1.7 \pm 1.3$ AIA31240-positive cells per section for Ad-LacZ, $P=0.76$, Table 1). Subendothelial presence of AIA31240positive cells in this part of the vessel wall was not detected.

Ad-FasL and Ad-LacZ-incubated arteries had a continuous CD31-positive lining with equal staining intensity both at the site of the lesion (Fig. 2I and J), as well as at the site of the non-diseased vessel (not shown), indicating that Ad-FasL did not affect the integrity of the endothelial lining.

\subsection{Effects of Ad-FasL three days after transfection}

Three days after FasL overexpression presence of AIA31240-positive cells subendothelial and at the lumenal side of the endothelial lining was strongly reduced and only observed in one out of nine plaques. Interestingly, in one plaque, we observed presence of cells, morphologically identified as red blood cells, suggesting intra-plaque hemorrhage. Detailed morphometrical analysis revealed no differences at three days after incubation between Ad-FasL and Ad-LacZ-treated carotid artery lesions for total intima area, macrophage area, cap-plaque ratio or cap-core ratio (Table 2). A tendency towards a $40 \%$ decrease in the cap SMC area was observed that coincided with a significant $38 \%$ reduction in number of cap cells (Table 2).

\subsection{Effects of Ad-FasL two weeks after transfection}

Total cross-sectional intima area did not differ between treatment groups (Table 3). The plaques of both groups consisted of a distinctive fibrous cap overlying a hypocellular core. The core and cap area and consequently also the cap-core ratio were not different between the treatment groups. In addition, the cap-intima ratio, the surface area of collagen-rich matrix as determined by Masson's trichrome and the average number of nuclei contained within each $5 \mu \mathrm{m}$ section of the cap were not different (Table 3). Intimal macrophage area was unaffected by Ad-FasL (Table 3). Staining for SM- $\alpha$-actin was confined to the media and the fibrous cap in both groups (not shown) and was not different between the treatment groups (Table 3).

\subsection{Evidence of rupture-related events in Ad-FasL-treated plaques}

Despite the absence of morphometrical differences two weeks after incubation, further histological analysis of the standard Masson's trichrome stained sections of the AdFasL-treated collar-induced plaques revealed the occurrence

Table 2

Morphometric evaluation of collar-induced atherosclerotic lesions of Ad-FasL-treated and contralateral Ad-LacZ-treated carotid arteries three days after transfection $(n=9)$

\begin{tabular}{|c|c|c|c|}
\hline Parameters & Ad-LacZ & Ad-FasL & $P$-value \\
\hline Media area $\left(\times 10^{3} \mu \mathrm{m}^{2}\right)$ & $47.5 \pm 7.5$ & $46.3 \pm 15.3$ & 0.906 \\
\hline Total intima area $\left(\times 10^{3} \mu \mathrm{m}^{2}\right)$ & $50.6 \pm 23.9$ & $40.5 \pm 15.1$ & 0.409 \\
\hline Macrophage area (from AIA31240) $\left(\times 10^{3} \mu \mathrm{m}^{2}\right)$ & $39.5 \pm 18.3$ & $33.7 \pm 13.4$ & 0.814 \\
\hline SMC cap area $($ SM- $\alpha$-actin $)\left(\times 10^{3} \mu \mathrm{m}^{2}\right)$ & $11.1 \pm 5.9$ & $6.7 \pm 2.7$ & 0.077 \\
\hline Cap-intima ratio & $0.2 \pm 0.04$ & $0.2 \pm 0.05$ & 0.110 \\
\hline Cap-core ratio & $0.3 \pm 0.06$ & $0.2 \pm 0.07$ & 0.087 \\
\hline Nuclei/cap & $111 \pm 37$ & $69 \pm 28$ & $0.013^{\mathrm{a}}$ \\
\hline Nuclei/cap surface area $\left(\times 10^{-3} \mu \mathrm{m}^{2}\right)$ & $12 \pm 6$ & $11 \pm 3$ & 1.000 \\
\hline
\end{tabular}

\footnotetext{
a Significantly different from Ad-LacZ-treated group.
} 
Table 3

Morphometric evaluation of collar-induced atherosclerotic lesions of Ad-FasL-treated and contralateral Ad-LacZ-treated carotid arteries 14 days after transfection $(n=13)$

\begin{tabular}{|c|c|c|c|}
\hline Parameters & Ad-LacZ & Ad-FasL & $P$-value \\
\hline Media area $\left(\times 10^{3} \mu \mathrm{m}^{2}\right)$ & $33.4 \pm 5.3$ & $36.8 \pm 9.2$ & 0.293 \\
\hline Total intima area $\left(\times 10^{3} \mu \mathrm{m}^{2}\right)$ & $44.1 \pm 26.2$ & $58.5 \pm 35.8$ & 0.343 \\
\hline Core area $\left(\times 10^{3} \mu \mathrm{m}^{2}\right)$ & $33.0 \pm 17.6$ & $48.1 \pm 34.0$ & 0.228 \\
\hline Macrophage area (from AIA31240) $\left(\times 10^{3} \mu \mathrm{m}^{2}\right)$ & $29.2 \pm 19.6$ & $41.8 \pm 27.9$ & 0.157 \\
\hline Cap area (collagen, Masson's) $\left(\times 10^{3} \mu \mathrm{m}^{2}\right)$ & $12.0 \pm 11.4$ & $11.3 \pm 7.1$ & 0.525 \\
\hline SMC cap area $(\mathrm{SM}-\alpha$-actin $)\left(\times 10^{3} \mu \mathrm{m}^{2}\right)$ & $10.5 \pm 6.2$ & $12.6 \pm 5.7$ & 0.327 \\
\hline Cap-intima ratio & $0.2 \pm 0.1$ & $0.2 \pm 0.1$ & 0.908 \\
\hline Cap-core ratio & $0.3 \pm 0.2$ & $0.3 \pm 0.1$ & 0.419 \\
\hline Nuclei/cap & $70 \pm 52$ & $86 \pm 42$ & 0.140 \\
\hline Nuclei/cap surface area $\left(\times 10^{-3} \mu \mathrm{m}^{2}\right)$ & $8 \pm 2$ & $8 \pm 3$ & 0.577 \\
\hline
\end{tabular}

of cap breaks in 2 of a total of 17 carotid arteries (Fig. 3A and B, Table 4), which was accompanied by infiltrated red blood cells not only at the shoulder regions, but also throughout the Ad-FasL-treated plaque, indicating intraplaque hemorrhage (IPH) had occurred. In addition, two cases of single buried caps were found, which is considered a sign of previous rupture [21]. Another two plaques showed IPH, of which one also had a buried cap. Both were confirmed by evidence of phagocytosis of erythrocytes by macrophages, as demonstrated by

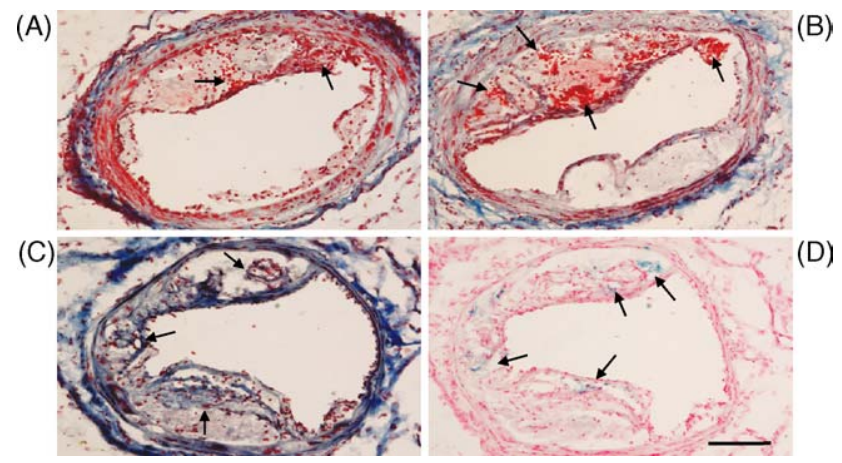

Fig. 3. Masson's trichrome (A, B, C) and Perl iron (D) stain demonstrating rupture-related events only in the Ad-FasL-treated carotid arteries 14 days after transfection of pre-existing carotid atherosclerosis. (A and B) Intraplaque hemorrhage (IPH). (C) IPH and a buried cap. (D) Serial section to $\mathrm{C}$ showing iron deposits (blue staining). Magnification 100×; scale bar $100 \mu \mathrm{m}$.

\section{Table 4}

Incidence of histological evidence of rupture-related events in Ad-FasLtreated and contralateral Ad-LacZ-treated carotid arteries $(n=17) 14$ days after adenovirus instillation

\begin{tabular}{lll}
\hline Event & \multicolumn{2}{l}{$\begin{array}{l}\text { Number of carotid arteries } \\
\text { with events }\end{array}$} \\
\cline { 2 - 3 } & Ad-LacZ & Ad-FasL \\
\hline Cap break + IPH & $0 / 17$ & $2 / 17$ \\
Buried caps & $0 / 17$ & $2 / 17$ \\
IPH + buried cap + iron deposits & $0 / 17$ & $1 / 17$ \\
IPH + iron deposits & $0 / 17$ & $1 / 17$ \\
Total & $0 / 17$ & $6 / 17$ \\
Fisher's exact test & \multicolumn{2}{c}{$P=0.009$} \\
\hline
\end{tabular}

Frequency data were compared by means of the Fisher's exact test.
Perls iron staining for the presence of macrophage processed iron (Fig. 3C and D). In total, 11 rupture-related events were detected in 17 Ad-FasL-treated carotid arteries. Evidence of rupture-related events was not found in the contralateral AdLacZ-treated control arteries $(n=17)$. The number of arteries displaying events in the Ad-FasL-treated group was statistically significant ( 6 out of 17 carotid arteries) as compared to the control Ad-LacZ-treated group ( 0 out 17; Fisher's exact test, $P=0.009$, Table 4 ).

\section{Discussion}

The presence of Fas and Fas ligand in human atherosclerotic plaques $[3,6]$, and the fact that human blood-derived macrophages can induce apoptosis in human plaque-derived SMCs by Fas/Fas ligand interactions in vitro [11], have suggested the Fas/Fas ligand pathway of apoptosis as a potential key player in lesion remodelling. In the present in vivo study we transfected the SMC-rich cap of pre-existing plaques with adenovirus carrying FasL. FasL expression led to a three-fold increase in cap-apoptosis one day after gene transfer. Three days after gene transfer, FasL-induced a $38 \%$ reduction in the number of cap cells. Two weeks after Ad-FasL transfer, non-thrombotic rupture, intra-plaque haemorrhage, buried caps and iron deposits were observed in 6 out of 17 Ad-FasL-treated carotid arteries versus 0 out of 17 contralateral Ad-LacZ-treated controls $(P=0.009)$, indicative of enhanced plaque vulnerability. We demonstrated that pre-existing murine plaques indeed are sensitive to the Fas/FasL apoptotic trigger. This might indicate that stimulation of this Fas/FasL pathway could result in plaque remodelling towards a more vulnerable phenotype.

In the current study, we investigated whether FasL is able to induce remodelling of advanced atherosclerotic plaques. Other in vivo studies on the role of FasL in vascular disease have used various models and gene expression protocols. In a non-injury hypercholesterolemic rabbit model, endothelial cells of non-atherosclerotic carotid arteries were transduced with FasL, resulting in decreased $\mathrm{T}$ cell infiltration and accelerated atherosclerotic lesion growth by increasing smooth 
muscle lesion cellularity [14]. Adenovirus-mediated delivery of FasL to denuded vessels caused inhibition of neointima formation in a rat carotid artery balloon-injury model in combination with decreased T-cell infiltration of the lesion [12]. Transgenic mice on an ApoE-/- background, that specifically overexpress different levels of FasL on vascular ECs, show reduced atherosclerotic lesion area in aortas. This coincided with decreases in both macrophage and CD8 T-cell accumulation in lesions [22]. In a rat aortic allograft model, adenovirus-mediated overexpression of soluble Fas blocked Fas binding to FasL and reduced CD45+ cell infiltration, thus protecting the integrity of the vessel wall from immune injury and attenuating transplant arteriosclerosis [23]. Overall, these studies using various models of vascular disease and gene expression protocols, suggest that Fas ligand functions to inhibit infiltration of inflammatory cells and thereby modulates the initiation and progression of the disease. In contrast, the present in vivo study focuses on pre-existing lesions and the SMC-rich cap is targeted. In line with in vitro findings $[7,9,11,24,25]$, we demonstrate that FasL is capable of inducing lesional apoptosis and thereby secondary recruitment of AIA31240-positive cells (monocytes/macrophages) was caused only at the level of the diseased atherosclerotic vessel wall. Apparently, Fas/FasL interactions result in various effects depending on the model and targeted cell type.

The procedure of local adenovirus instillation allows FasL gene delivery to the superficial layer ,i.e. cap of the plaque. Indeed, TUNEL-positive cells located in an area stained positive for SM- $\alpha$-actin (Fig. 2E and F). Due to antigen loss, the double SM- $\alpha$-actin- TUNEL staining was weak. However, together this suggests that SMC apoptosis, at least in part, underlies the observed increased vulnerability of the atherosclerotic plaque. This is in line with the in vitro finding that (macrophage) FasL can induce apoptosis in human plaquederived smooth muscle cells [11]. In addition to SMCs, ECs and superficial macrophages can be targeted by our local adenovirus procedure. On the other hand, macrophages are known to be very hard to transduce in vivo [26]. Whereas, ECs do express Fas on their cell surface under normal, but also pathological conditions, they are resistant to Fas-mediated apoptosis $[14,27,28]$. This was supported by our observation that FasL did not affect the integrity of the continuous CD31-positive endothelial lining covering the plaque. In addition to SMC apoptosis, macrophage foam cells play a key role in the vulnerability of the atherosclerotic plaque. A high macrophage:SMC ratio makes a plaque more prone to rupture. Previous studies have shown that macrophages and SMCs in the atherosclerotic plaque synthesize and secrete matrix proteases, like metalloprotease 1 and 3 [29,30]. These MMP's can degrade the fibrous cap and thereby contribute to plaque instability. Although there is no difference in the number of macrophages in the FasL and control-treated lesions, we cannot exclude the possibility that the lesion specific macrophages may in fact differ in their propensity to induce plaque rupture.
In human vascular SMCs, p53 can mediate apoptosis through Fas transport from cytoplasmic stores and increase sensitivity to FasL-induced death [9]. Possibly, p53-induced lesion destabilization and rupture in murine lesions also involves the Fas/FasL pathway. In a previous study, we indeed demonstrated that $\mathrm{p} 53$-induced destabilization and rupture of pre-existing collar-induced atherosclerotic plaques of ApoEdeficient mice, which was, at least in part, due to the induction of apoptosis [17]. Induction of rupture-related events by p53 however, was observed only after vassopressor treatment of the mice one day before sacrifice. Although p53 and FasL share molecular pathways through which they induce apoptosis, major differences exist between the two proteins, which could explain the observed differences between p53 and FasL-treated plaques. P53 is a highly regulated transcription activator inducing expression of numerous genes, many of which not related to apoptosis. FasL, on the other hand, acts predominantly as a fast and potent killer of Fas-expressing cells. Interestingly, Fas activation has been reported to induce the expression of a variety of genes encoding inflammatory proteins in carotid artery SMCs [31]. Increased inflammatory gene expression may underlie the increased recruitment of AIA31240-positive cells (monocytes/macrophages) observed for FasL and not for p53. Detailed studies on the mechanism underlying the observed differences between $\mathrm{p} 53$ and FasL may help to identify factors involved in plaque destabilisation.

Rupture-prone plaques exhibit accumulation of various pro-inflammatory cytokines from T-cells, monocytes/ macrophages, as well as SMCs [31]. In vitro it was shown that only after treatment with cytokines, such as interferon gamma (IFN $\gamma$ ), interleukin- $1 \beta$, or TNF- $\alpha$, which are also present in the plaque, SMC were sensitive to Fas-mediated death [6]. In addition, pharmacological concentrations of some statins have also been found to "sensitize" SMCs to Fas-mediated death [32]. Our model, in which rupture occurs more frequently and in a controlled fashion, may help to delineate the molecular pathways involved in plaque (de)stabilization and to evaluate (anti-inflammatory) therapies aimed at plaque stabilization and prevention.

Our p53 and FasL adenovirus studies were both aimed at triggering endogenous pathologically activated pathways in the atherosclerotic plaque. In both studies, we observed only a limited increase in apoptosis (up to $1.5 \%$ of cap cells) already coinciding with destabilization of the plaque and rupturerelated events. Our experimental approach using transgenic mice with collar-induced lesions and adenovirus mediated gene delivery may be far from the human and physiologically relevant situation. However, the p53 or FasL-induced apoptosis levels are still within the range observed in human atherosclerotic lesions [18]. Together with human histological data [3-5] and in vitro studies with human cells [9-11,24,25], our data suggest that p53 and Fas may be important and physiologically relevant triggers for apoptosis and remodelling of the plaque towards a more vulnerable phenotype. 


\section{Acknowledgments}

This study was supported by NWO/ ZonMw grant nos. 902-26-242 (BJMvV) and 912-02-03 (BJMvV, EALB), by grant nos. 2000.051 and M93.001 (JHvdT) of the Netherlands Heart Foundation. The research of Dr. B.J.M. van Vlijmen has been made possible by a fellowship of the Royal Netherlands Academy of Arts and Sciences. We thank Martijn J.W.E. Rabelink (Department of Molecular Cell Biology, Leiden University Medical Center, Leiden, The Netherlands), Corine van Helden-Meeuwsen and Tar van Os (Department of Immunology, Erasmus MC, Rotterdam, The Netherlands) for their excellent technical assistance.

\section{References}

[1] Nagata S, Golstein P. The Fas death factor. Science 1995;267: 1449-56.

[2] Bellgrau D, Duke RC. Apoptosis and CD95 ligand in immune privileged sites. Int Rev Immunol 1999;18:547-62.

[3] Cai W, Devaux B, Schaper W, Schaper J. The role of Fas/APO 1 and apoptosis in the development of human atherosclerotic lesions. Atherosclerosis 1997;131:177-86.

[4] Henderson EL, Geng YJ, Sukhova GK, et al. Death of smooth muscle cells and expression of mediators of apoptosis by T lymphocytes in human abdominal aortic aneurysms. Circulation 1999;99:96-104.

[5] Kiener PA, Davis PM, Rankin BM, et al. Human monocytic cells contain high levels of intracellular Fas ligand: rapid release following cellular activation. J Immunol 1997;159:1594-8.

[6] Geng YJ, Henderson LE, Levesque EB, Muszynski M, Libby P. Fas is expressed in human atherosclerotic intima and promotes apoptosis of cytokine-primed human vascular smooth muscle cells. Arterioscler Thromb Vasc Biol 1997;17:2200-8.

[7] Sata M, Suhara T, Walsh K. Vascular endothelial cells and smooth muscle cells differ in expression of Fas and Fas ligand and in sensitivity to Fas ligand-induced cell death: implications for vascular disease and therapy. Arterioscler Thromb Vasc Biol 2000;20:30916.

[8] Isner JM, Kearney M, Bortman S, Passeri J. Apoptosis in human atherosclerosis and restenosis. Circulation 1995;91:2703-11.

[9] Bennett M, Macdonald K, Chan SW, et al. Cell surface trafficking of Fas: a rapid mechanism of p53-mediated apoptosis. Science 1998;282:290-3.

[10] Yao PM, Tabas I. Free cholesterol loading of macrophages induces apoptosis involving the fas pathway. J Biol Chem 2000;275:23807-13.

[11] Boyle JJ, Bowyer DE, Weissberg PL, Bennett MR. Human bloodderived macrophages induce apoptosis in human plaque-derived vascular smooth muscle cells by Fas-ligand/Fas interactions. Arterioscler Thromb Vasc Biol 2001;21:1402-7.

[12] Luo Z, Sata M, Nguyen T, et al. Adenovirus-mediated delivery of fas ligand inhibits intimal hyperplasia after balloon injury in immunologically primed animals. Circulation 1999;99:1776-9.

[13] Sata M, Perlman H, Muruve DA, et al. Fas ligand gene transfer to the vessel wall inhibits neointima formation and overrides the adenovirus-mediated $\mathrm{T}$ cell response. Proc Natl Acad Sci USA 1998;95:1213-7.

[14] Schneider DB, Vassalli G, Wen S, et al. Expression of Fas ligand in arteries of hypercholesterolemic rabbits accelerates atheroscle- rotic lesion formation. Arterioscler Thromb Vasc Biol 2000;20:298308.

[15] der Thusen JH, van Berkel TJ, Biessen EA. Induction of rapid atherogenesis by perivascular carotid collar placement in apolipoprotein E-deficient and low-density lipoprotein receptor-deficient mice. Circulation 2001;103:1164-70.

[16] Fallaux FJ, Bout A, van dV I, et al. New helper cells and matched early region 1-deleted adenovirus vectors prevent generation of replication-competent adenoviruses. Hum Gene Ther 1998;9:1909-17.

[17] der Thusen JH, van Vlijmen BJ, Hoeben RC, et al. Induction of atherosclerotic plaque rupture in apolipoprotein $\mathrm{E}-/-$ mice after adenovirus-mediated transfer of p53. Circulation 2002;105:2064-70.

[18] Kockx MM. Apoptosis in the atherosclerotic plaque: quantitative and qualitative aspects. Arterioscler Thromb Vasc Biol 1998;18:1519-22.

[19] Gavrieli Y, Sherman Y, Ben Sasson SA. Identification of programmed cell death in situ via specific labeling of nuclear DNA fragmentation. J Cell Biol 1992;119:493-501.

[20] Van Blokland SC, Helden-Meeuwsen CG, Wierenga-Wolf AF, et al. Apoptosis and apoptosis-related molecules in the submandibular gland of the nonobese diabetic mouse model for Sjogren's syndrome: limited role for apoptosis in the development of sialoadenitis. Lab Invest 2003;83:3-11.

[21] Williams H, Johnson JL, Carson KG, Jackson CL. Characteristics of intact and ruptured atherosclerotic plaques in brachiocephalic arteries of apolipoprotein E knockout mice. Arterioscler Thromb Vasc Biol 2002;22:788-92.

[22] Yang J, Sato K, Aprahamian T, et al. Endothelial overexpression of fas ligand decreases atherosclerosis in apolipoprotein e-deficient mice. Arterioscler Thromb Vasc Biol 2004;24:1466-73.

[23] Wang T, Dong C, Stevenson SC, et al. P.J. Overexpression of soluble fas attenuates transplant arteriosclerosis in rat aortic allografts. Circulation 2002;106:1536-42.

[24] Bennett MR, Evan GI, Schwartz SM. Apoptosis of human vascular smooth muscle cells derived from normal vessels and coronary atherosclerotic plaques. J Clin Invest 1995;95:2266-74.

[25] Chan SW, Hegyi L, Scott S, et al. Sensitivity to Fas-mediated apoptosis is determined below receptor level in human vascular smooth muscle cells. Circ Res 2000;86:1038-46.

[26] Burke B, Sumner S, Maitland N, Lewis CE. Macrophages in gene therapy: cellular delivery vehicles and in vivo targets. J Leukoc Biol 2002;72:417-28.

[27] Tran TH, Grey S, Anrather J, et al. Regulated and endothelial cell-specific expression of Fas ligand: an in vitro model for a strategy aiming at inhibiting xenograft rejection. Transplantation 1998;66:1126-31.

[28] Sata M, Luo Z, Walsh K. Fas ligand overexpression on allograft endothelium inhibits inflammatory cell infiltration and transplantassociated intimal hyperplasia. J Immunol 2001;166:6964-71.

[29] Galis ZS, Sukhova GK, Lark MW, Libby P. Increased expression of matrix metalloproteinases and matrix degrading activity in vulnerable regions of human atherosclerotic plaques. J Clin Invest 1994;94:2493-503.

[30] Galis ZS, Muszynski M, Sukhova GK, et al. Cytokine-stimulated human vascular smooth muscle cells synthesize a complement of enzymes required for extracellular matrix digestion. Circ Res 1994;75:181-9.

[31] Schaub FJ, Han DK, Liles WC, et al. D.F. Fas/FADD-mediated activation of a specific program of inflammatory gene expression in vascular smooth muscle cells. Nat Med 2000;6:790-6.

[32] Knapp AC, Huang J, Starling G, Kiener PA. Inhibitors of HMGCoA reductase sensitize human smooth muscle cells to Fas-ligand and cytokine-induced cell death. Atherosclerosis 2000;152:217-27. 